BACK GROUND: Endometriosis is defined as an inflammatory condition of the female reproductive tract, a state often associated with infertility and miscarriage. Many exogenously administered factors (treatments) control the disease via as yet unknown pathways. Possible candidate molecules involved in these mechanisms could be the serum-soluble human leukocyte antigens (sHLA) that have been detected in a variety of human body fluids and that are associated with several diseases.

Aims: We here examine how danazol and leuprorelin acetate depot treatments exert their anti-inflammatory action. It is plausible that subtle alterations mediated by these treatments and in relation to sHLA may explain the pathophysiology of endometriosis and provide insights towards new therapeutic protocols.

Metbods: Indirect enzyme-linked immunosorbent assay (ELISA), using specific monoclonal antibodies, determined serum-soluble class-I and class-II HLA levels. ELISA readings from treated women were compared with normal healthy subjects.

Results: Serum-soluble class-I and class-II HLA levels are statistically significantly lower $(P<0.001)$ in women with endometriosis than in the control groups. However, danazol but not leuprorelin acetate depot administration augments soluble HLA class I and class II $(P<0.01$ and $P<0.001$, respectively) to normal levels during the treatment period, an increase that may account for the anti-inflammatory effect and the remission observed.

Conlusions: It is shown that one of the underlying causes of endometriosis may be the lack of both circulating class-I and class-II antigen levels. Danazol administration acts via an induced release of these antigens, whose presence correlates with the degree of the inflammatory alleviation obtained. We thus provide evidence that the inflammatory state of the disease appears to be associated with soluble HLA levels because, 3 months after ceasing therapy, the circulating antigens in the serum return to the same levels that correspond to the pathological condition.

Key words: Endometriosis, Danazol, Leuprorelin acetate depot, Circulating antigens, Soluble HLA class I, Soluble HLA class II

\section{The possible anti-inflammatory role of circulating human leukocyte antigen levels in women with endometriosis after treatment with danazol and leuprorelin acetate depot}

loannis M. Matalliotakis ${ }^{1}$, Irene Athanassakis ${ }^{2}$, Anastasia G. Goumenou ${ }^{1}$, Maria A. Neonaki ${ }^{1}$, Emmanuil E. Koumantakis ${ }^{1}$, Simon Vassiliadis ${ }^{1,2, C A}$ and Evgenios E. Koumantakis ${ }^{1}$

${ }^{1}$ Department of Obstetrics and Gynecology, University Hospital, University of Crete, Heraklion, Greece, and ${ }^{2}$ Department of Biology, University of Crete, P.O. Box 2208, Heraklion 714-09, Greece

\footnotetext{
${ }^{\mathrm{CA}}$ Corresponding Author

Tel: +30 81394355

Fax: +3081394379

E-mail: simon@biology.uoc.gr
}

\section{Introduction}

Endometriosis has been well described and known for over 50 years. However, our current knowledge of the pathogenesis, pathophysiology of related infertility and spontaneous evolution remains unclear.

One theory for the development of endometriosis suggests an aberrant immune system as a causative factor. Studies have demonstrated that women with endometriosis have altered cell-mediated immunity, ${ }^{1-3}$ which could allow translocated endometrial cells to implant and progress. Additionally, women with endometriosis have also been shown to exhibit an altered humoral immunity ${ }^{4-6}$ as well as increased macrophage numbers and their activation, ${ }^{7}$ events that lead to a severe local inflammatory state.

Surface human leukocyte antigen (HLA) genotypes have been associated with more than 30 diseases, including systemic lupus erythomatosus, ankylosing spondylitis, and multiple sclerosis. The etiology and pathogenesis of many of these, mainly autoimmune, disorders are unknown. Susceptibility to diseases associated with surface HLA is likely to be determined by multiple genes as well as environmental factors. ${ }^{8}$ 
The complexity of endometriosis and the suggestion of an autoimmune basis underlying this particular malady led investigators to evaluate the HLA genotype in endometriosis. ${ }^{9,10}$ Moen et al. ${ }^{10}$ studied surface HLA in 100 women with endometriosis where no association between HLA and the disease was found.

Recently, however, it has been shown that intracellular pools of HLA, residing in cytoplasmic organelles, may be released to the extracellular matrix as soluble particles, and may enter the circulation following stimulation by various endogenous or exogenous factors. ${ }^{11}$ Serum-soluble (circulating) HLA antigens have also been detected in a variety of human body fluids and associated with several diseases (reviewed in Ref. 12). Candidate stimuli may be considered a wide array of factors that have been reviewed by Ho et al. ${ }^{13}$ and comprise known inflammatory cytokines, including interferon- $\gamma$ (IFN$\gamma$ ) and tumor necrosis factor- $\alpha$ (TNF- $\alpha$ ), characterized by their ability to induce surface histocompatibility antigens. Since endometriosis is defined as an inflammatory condition of the female reproductive tract, and occurrence of both IFN- $\gamma$ and TNF- $\alpha$ has been documented in this particular state, ${ }^{13}$ we inquired whether secreted/soluble HLA class-I and class-II antigens are present in the serum of endometriotic patients. We also investigated the effect of danazol and leuprorelin acetate depot on the levels of these antigens, and correlated the findings to their pharmaceutical anti-inflammatory action.

\section{Materials and methods}

\section{Patients}

The study population included 68 women with infertility that attended the Department of Obstetrics and Gynecology of the University Hospital of Crete from 1991 to 1999. All the women underwent laparoscopic examination because of suspected endometriosis. The University Committee approved specific and future protocols, and written informed consent was obtained from each subject before participation, all in accordance with the Declaration of Helsinki. Sera of the women, who accepted to be part of the research projects, have been previously used for the detection of other immunologic variables $^{5,6}$ as well as prolactin, thyroid stimulating hormone and soluble CD23. ${ }^{14,15}$

Thirty-eight of the women had endometriosis (mean, $27.7 \pm 5.8$ years) and 30 had no pelvic disease (mean, $29.2 \pm 5.7$ years). After their case histories were compiled, clinical examinations were carried out; their husbands' semen was normal in all cases. Thereafter, ovulation was tested, cervical mucus evaluated, and tubal potency ascertained by hysterosalpingography. All examinations showed normal results for all women.
According to the classification issued by the American Fertility Society, ${ }^{16}$ subjects were classified into two groups: 30 with pelvic pain who had no disease on laparoscopy were considered as controls, and 38 women with laparoscopically diagnosed and staged endometriosis (15 cases) were at stage I, 11 at stage II, six at stage III, and six at stage IV. Due to the diversity of the aforementioned endometriotic stages, the patients underwent different extents of surgery. No residues of endometriotic tissue could be detected macroscopically after completion of surgery. This observation assures that our results are free of any possible bias that would jeopardize the interpretation of the results that follow. The study was open-labeled and the women with endometriosis were assigned to the two groups (danazol and leuprorelin).

The first 11 women were given danazol capsules, an isoxazolic derivative of synthetic steroid, 17 a-ethynyletestosterone, $200 \mathrm{mg}$ by mouth every $8 \mathrm{~h}$ for 6 months. The stage of endometriosis of this specific group of study was in favor of stage I since five out of the 11 patients were in this category. The group also consisted of two women in each of the II, III and IV stages.

The second group of 11 women was treated with leuprorelin acetate depot, $3.75 \mathrm{mg}$ every 28 days for 6 months. This set contained three patients in stage I, four in stage II, and two in each of the III and IV stages.

Finally, the distribution stage of endometriotic women who did not receive treatment (16 patients) was seven in stage I, five in II, and two in each of the III and IV stages. Blood samples were taken from all women before laparoscopy for the evaluation of soluble HLA class-I and class-II levels.

Serum-soluble HLA levels were also measured in the 22 endometriotic women, before treatment, during the last 15 days of a 6-month course of danazol and leuprorelin acetate, and 3 months after treatment. Blood samples from women with endometriosis were collected between the fifth and seventh day of their menstrual cycle before and after treatment. Within $1 \mathrm{~h}$, blood samples were centrifuged at $3000 \mathrm{rpm}$ (400 $\times g$ ) and serum was kept at $-80^{\circ} \mathrm{C}$ until assayed.

\section{Reagents}

Serum-soluble HLA (class I and class II) levels were determined by indirect enzyme-linked immunosorbent assay (ELISA) ${ }^{17,18}$ (see next section), using the monoclonal antibodies 83H1 (HLA-A,B,C; IgG1) and 7 H3 (HLA-DR; IgG2a), respectively. ${ }^{19,20}$

\section{Enzyme-linked immunoassay (ELISA)}

The ELISA experiments were performed as previously described. ${ }^{17,18}$ First, in order to ensure linearity between antigen concentration (sera) and optical 


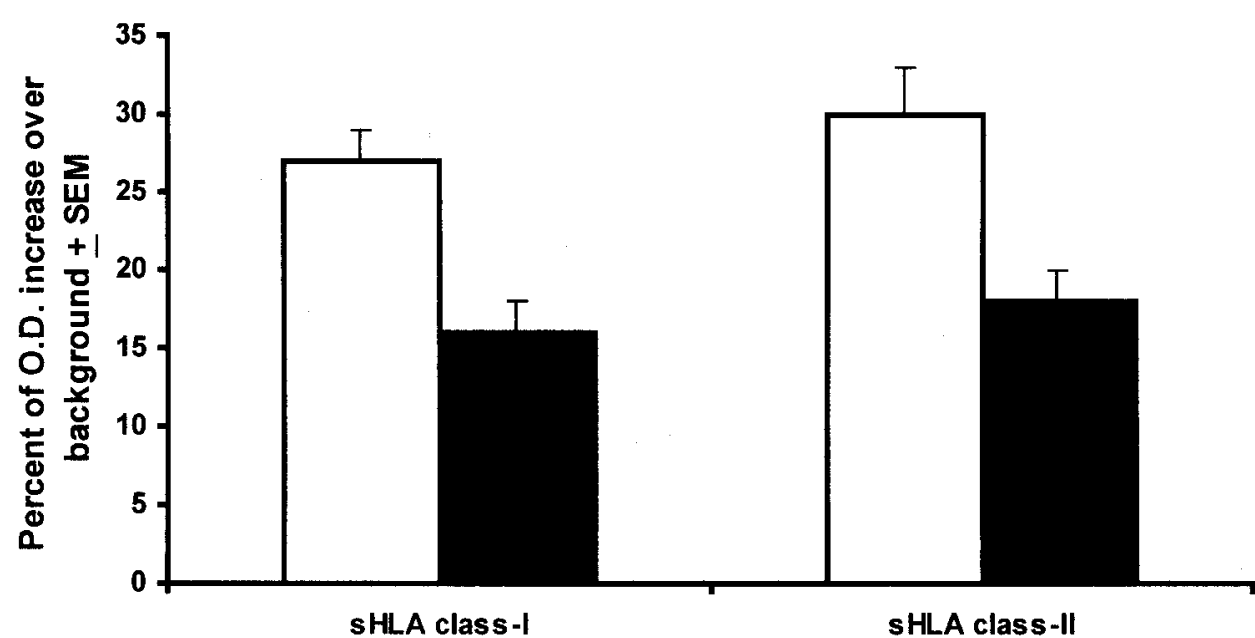

FIG. 1. Serum-soluble human leukocyte antigen (sHLA) class-I and class-II levels in healthy subjects (controls, white bars) and in women with endometriosis (black bars). Soluble class I was detected by the monoclonal antibody 83H1 (HLA-A,B,C, $\operatorname{lgG} 1)^{19,20}$ and class II by the monoclonal antibody $7 \mathrm{H} 3$ (HLA-DR, IgG2a). ${ }^{20}$ The decrease of soluble antigens for both HLA classes is of the significance order of $P<0.001$. The results are expressed as percent of optical density (OD) increase over background levels $( \pm S E M)$. Background levels (OD values of coating buffer) corresponded to $0.110 \pm 0.015$ and $0.075 \pm 0.008$ for the class-I and class-II antibodies, respectively. The experiment was repeated three times and similar results were obtained.

density (OD) correlation, we tested our samples at various dilutions $(1: 10,1: 50,1: 100,1: 500$ and 1:100) and chose the appropriate one. Briefly, sera collected as already mentioned were used at a concentration of 1:100 in carbonate buffer ( $\mathrm{pH}$ 9.6). These were coated in 96-well flat bottom plates (Sarstedt, Numbrecht, Germany), incubated overnight at $4^{\circ} \mathrm{C}$ and washed four times in 5\% Tween-20 (Sigma, St. Louis, MO, USA). The remaining protein free sites in the plate were blocked by $2 \%$ phosphate-buffered saline-bovine serum albumen (PBS-BSA) solution after an incubation of $2 \mathrm{~h}$ at room temperature. After washing four times, $100 \mu 1$ test antibody diluted in $0.1 \%$ PBS-BSA were added and incubated for $1 \mathrm{~h}$ at room temperature. Extensive washing of the plate was followed by addition of $100 \mu 1$ goat anti-rabbit IgG coupled to horseradish peroxidase (1:1000 dilution; Sigma) and incubated for $1 \mathrm{~h}$ at room temperature, in the dark. Finally, the reaction was developed by adding $100 \mu \mathrm{l} /$ well tetramethyl benzidine- $\mathrm{H}_{2} \mathrm{O}_{2}$ (Sigma) for $20 \mathrm{~min}$. The enzymatic reaction was stopped with $50 \mu 1 \mathrm{H}_{2} \mathrm{SO}_{4}(4 \mathrm{~N})$. OD was measured at $450 \mathrm{~nm}$ using a Titertec ELISA photometer (Digiscan; ASYS Hitech $\mathrm{GmbH}$, Engendorf, Austria). All samples from each of the women were measured in the same run and the results were expressed as the percent of optical density increase over background, i.e. test's internal control $( \pm$ SEM).

\section{Statistical analysis}

The paired Student $t$-test was used for statistical evaluation. For statistical analysis, we used an independent $t$-test for among-group comparisons, and the paired $t$-test for within-group comparisons, and the
Pearson correlation coefficient for correlation analysis.

\section{Results}

The percent of serum-soluble HLA class-I and class-II levels in women without endometriosis (controls) and those with endometriosis are presented in Figure 1. As this figure shows, soluble HLA (sHLA) class-I and class-II levels (percent of OD increase over background) were statistically significantly lower $(P<$ 0.001 ) in women with endometriosis than in control subjects.

All 22 patients developed amenorrhea during the danazol and leuprorelin acetate depot treatments. The before-treatment menstrual cycles of women studied were normal. As expected, pain related to endometriosis was gradually alleviated and menses disappeared in all 22 women during treatment. After cessation of treatment, menstrual bleeding returned to normal in 1-2 months. The percent of modulation of the serum-soluble HLA class I in the women with endometriosis before, during and 3 months after treatment with danazol or leuprorelin, respectively, are presented in Figure 2. As shown in this figure, sHLA class-I levels were statistically significantly higher $(P<0.01)$ during danazol treatment than before treatment. On the contrary, leuprorelin acetate depot administration had no effect on the levels of sHLA class I (Fig. 2). Three months after ceasing the treatments, sHLA class I returned to almost the same level as before treatment (Fig. 2).

Figure 3 provides the serum levels expressed as percent of OD increase over background (mean \pm SEM) of the sHLA class II in women with endome- 


\section{Soluble HLA class-I}

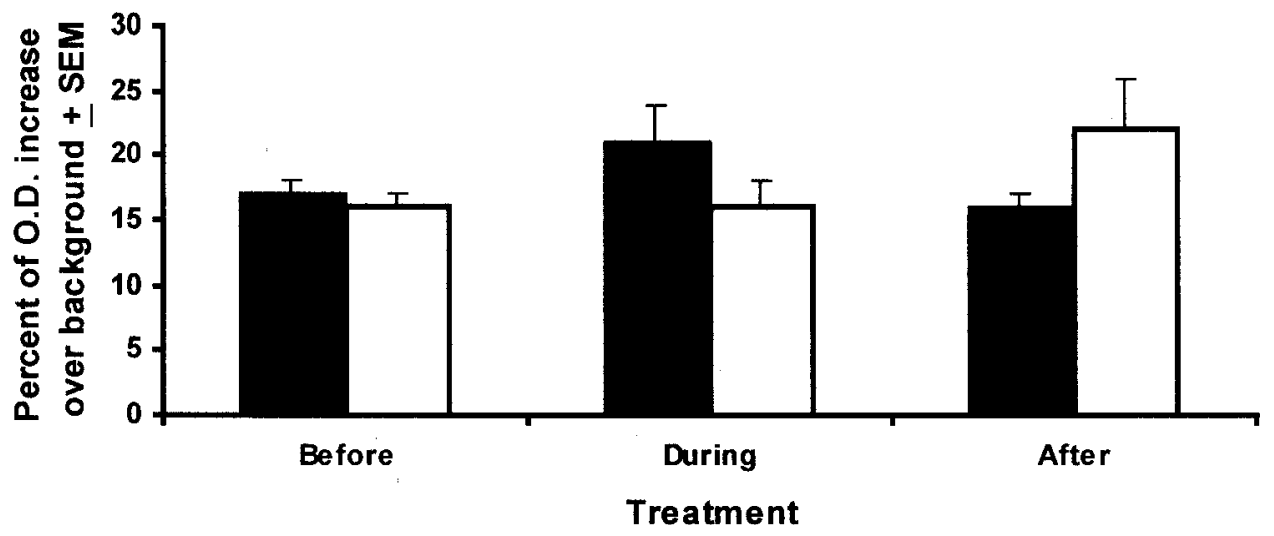

FIG. 2. Serum-soluble human leukocyte antigen (sHLA) class-I levels in women with endometriosis before, during and after treatment with danazol (black bars) or leuprorelin acetate depot (white bars), respectively. For the antibody employed for class I, see Figure 1. The increase obtained in response to danazol during the actual treatment time is of the order of $P<0.01$. The results are expressed as the percent of optical density (OD) increase over background levels $( \pm S E M)$. Background levels $(O D$ values of coating buffer) for the class-I antibody corresponded to $0.110 \pm 0.015$. The experiment was repeated three times and similar results were obtained.

triosis before, during and 3 months after treatment with danazol or leuprorelin, respectively. As shown in this figure, sHLA class-II levels were statistically significantly higher $(P<0.001)$ during danazol treatment than before treatment. In contrast, leuprorelin administration did not change significantly the levels of sHLA class II (Fig. 3). Three months after abandoning treatments, sHLA class-II levels reverted to those before treatment (Fig. 3). Finally, it is underlined that a correlation was not found between sHLA class I and class II and the severity (stage) of endometriosis.

\section{Discussion}

Several population and family studies have disclosed an association between certain diseases and a particular surface HLA phenotype. These diseases frequently manifest immunologic disorders. Population studies yield statistically strong evidence in support of an association between a disease susceptibility gene and a given HLA antigen/epitope. Through family studies, on the contrary, investigators can determine whether a linkage exists between HLA and a disease susceptibility gene.

\section{Soluble HLA class-II}

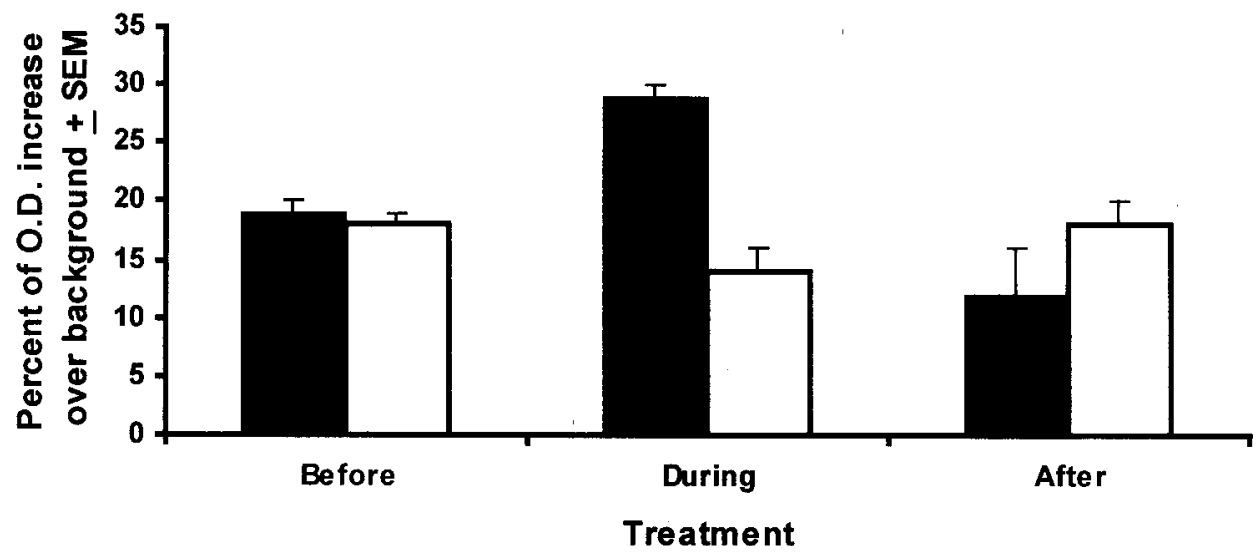

FIG. 3. Serum-soluble human leukocyte antigen (sHLA) class-II levels in women with endometriosis before, during and after treatment with danazol (black bars) or leuprorelin acetate depot (white bars), respectively. For the antibody employed for class II, see Figure 1. The increase obtained in response to danazol during the actual treatment time is of the order of $P<0.001$. The results are expressed as the percent of optical density (OD) increase over background levels $( \pm S E M)$. Background levels $(O D$ values of coating buffer) for the class-II antibody corresponded to $0.075 \pm 0.008$. The experiment was repeated three times and similar results were obtained. 
Endometriosis shows these characteristics. Simpson et al. ${ }^{21}$ demonstrated an increased incidence and severity of endometriosis in first-degree relatives of probands with endometriosis. Epidemiologic studies have also shown a familial tendency and an increased concordance of endometriosis in twins. ${ }^{22-25}$ Although these findings are consistent with inheritance of this disease as a polygenic/multifactorial trait, etiologic factors causing endometriosis remain largely unknown.

Surface HLA expression, an event under genetic control, and endometriosis appear to be independent findings since Moen et al. ${ }^{10}$ has demonstrated that there is no correlation between these two parameters. There are, however, intracellular stores containing HLA antigens that await a stimulus in order to be expressed on the surface and released in the extracellular matrix. These soluble/circulating HLA forms have been discovered in numerous body fluids, yet their significance remains speculative. ${ }^{12}$

Active secretion of HLA class I and class II by liver cells and activated immunocompetent cells has made these secreted forms be considered as normal constituents of the serum. ${ }^{12,26}$ In a recent study, the levels of the serum-soluble forms of class-I and class-II antigens have been established during normal pregnancy and first trimester abortions, ${ }^{27}$ and their presence has been correlated to a successful pregnancy outcome since they can be used as predictive markers. This observation renders the production/ presence of these molecules in the serum attractive candidates for the delineation of infertility in general. If one thus considers that sHLA-DR (class II) levels appear to be relatively high during normal pregnancy and, according to the present communication, sHLA class-II levels are lower than normal during endometriosis, it may be claimed that endometriosis-caused infertility is associated with the levels of serumsoluble HLA class-II antigens. By the same token, the extremely low levels of sHLA class I detected in first trimester abortions ${ }^{27}$ and their suppression in endometriotic women may imply that absence of this secreted molecule(s) is also involved in the pathways of infertility.

In the present study, we found that circulating HLA class-I and class-II levels are low in infertile women with endometriosis compared with controls. To date, no data are available concerning sHLA class-I and class-II levels during treatment with danazol and leuprorelin acetate depot in women with endometriosis. It is surprising that danazol but not leuprorelin administration increased significantly the levels of both soluble class I and class II $(P<0.01$ and $P<0.001$, respectively). This difference, however, shows the dissimilar mode of action of the two therapies and may underlie the fact that, pharmacologically, leuprorelin is less potent than danazol.
Three months after withdrawing the danazol or leuprorelin acetate depot treatments, the levels of sHLA class I and class II returned to almost the same as those before administration (Figs. 2 and 3). These results strongly indicate involvement of soluble/ circulating HLA antigens in the development and maintenance of ectopic endometrial cells.

The mechanism(s) by which an HLA antigen loses its ability to be anchored in the cell membrane and becomes released has recently gained grounds of evidence. ${ }^{11}$ There is increasing support, however, that the serum-soluble HLA forms play a role in the pathophysiology of many diseases that, to date, had no genetic link with specific surface HLA epitopes. ${ }^{28}$ This view renders the study of many maladies a complicated task to undertake since the regulatory mechanisms that govern such transition(s) will engage a number of new parameters that will delay the delineation of the pathways involved. In our laboratory, we are currently investigating how the circulating human leukocyte antigens affect endometriosis, and are attempting to determine the optimal levels to which an organism may be tolerant and thus unaffected by their presence so as to prevent the ailment.

ACKNOWLEDGEMENTS. The authors thank A. Ranella and L. Papadimitriou for technical assistance.

\section{References}

1. Dmowski WP, Steele RW, Baker GF Deficient cellular immunity in endometriosis. Am J Obstet Gynecol 1981; 141: 37-83.

2. Panidis DK, Matalliotakis IM. Subfertility associated with minimal to mild endometriosis. Main mechanisms. J Reprod Med 1998; 43: 1034-1042.

3. Oosterlynck DJ, Cornillie FJ, Waer M, Vandeputte M, Konincky PR. The natural killer activity of peritoneal fluid lymphocytes is decreased in women with endometriosis. Fertil Steril 1992; 58: 292-295.

4. Badawy SZ, Cuensa V, Stitzel A, Jacobs RB, Tomar RH. Autoimmune phenomena in infertile patients with endometriosis. Obstet Gynecol 1984; 63: 271-275.

5. Matalliotakis I, Neonaki M, Zolindaki A, Hassan E, Georgoulias V, Koumantakis E. Changes in immunologic variables (TNF- $\alpha$, sCD8 and sCD4) during danazol treatment in patients with endometriosis. Int J Fertil Women's Med 1997; 42: 211-214.

6. Koumantakis E, Matalliotakis I, Neonaki M, Froudarakis G, Georgoulias V. Soluble serum interleukin-2 receptor, interleukin- 6 and interleukin-1 $\alpha$ in patients with endometriosis and in controls. Arch Gynecol Obstet 1994 255: 107-112.

7. Halnes J, Becker S, Wing R. Accentuated cyclic activation of peritoneal macrophages in patients with endometriosis. Am J Obstet Gynecol 1984 148: 85-90.

8. Ross HL, Bischoff FZ, Elias S. Genetics of endometriosis. In: Diamond MP, Osteen KG, eds. Endometrium and Endometriosis. Boston, MA: Blackwell Science, 1997: 70-74.

9. Ota H, Igarashi S. Expression of major histocompatibility complex class II antigen in endometriotic tissue in patients with endometriosis and adenomyosis. Fertil Steril 1993; 60: 834-838.

10. Moen M, Bratlie A, Moen T. Distribution of HLA-antigens among patients with endometriosis. Acta Obstet Gynecol Scand 1984; 123 (Suppl) 25-27.

11. Athanassakis I, Ranela A, Vassiliadis S. IFN-gamma facilitates release of class II-loaded intracellular pools in trophoblast cells: a novel property independent of protein synthesis. J Interferon Cytokine Res 2000: 20: 823-830.

12. Aultman D, Adamashvili I, Yaturu K, Langford M, Gelder F, Gautreaux M, Ghali G, McDonald J. Soluble HLA in human body fluids. Hum Immunol 1999; 60: 239-244.

13. Ho HN, Wu MY, Yang YS. Peritoneal cellular immunity and endometriosis. Am J Reprod Immunol 1997; 38: 400-412.

14. Matalliotakis I, Panidis D, Vlassis G, Vavilis D, Neonaki M, Koumantakis E. PRL TSH and their response to the TRH test in patients with endometriosis before, during and after treatment with danazol. Gynecol Obstet Invest 1996; 42: 183-186. 
15. Matalliotakis I, Neonaki M, Koumantaki YG, Goumenou AG, Kyriakou DS, Koumantakis EE. A randomized comparison of danazol and leuprolide acetate suppression of serum-soluble CD23 levels in endometriosis. Obstet Gynecol 2000; 95: 810-813.

16. American Fertility Society. Revised American Fertility Society classification of endometriosis. Fertil Steril 1985; 43: 351-356.

17. Athanassakis I, Iconomidou B. Cytokine production in the serum and spleen of mice from day 6 to 14 of gestation: cytokines/placenta/spleen/ serum. Dev Immunol 1996; 4: 247-255.

18. Athanassakis I, Farmakiotis V, Papadimitriou L. Uterine cytokine production during the menstrual cycle and preimplantation stages in mice. Dev Immunol 1999; 7: 33-42.

19. Sutherland J, Mannoni P, Rosa F, Huyat D, Turner AR, Fellons M. Induction of the expression of HLA class I antigen or K562 by interferons and sodium butyrate. Hum Immunol 1985; 12: 65-73.

20. Vassiliadis S, Guilbert LJ. Immune interferon induces early and late events in differentiation of HL- 60 cells that are part of the same maturation program. Exp Hematol 1991; 19: 250-256.

21. Simpson JL, Elias S, Malinak LR, Buttram VC. Heritable aspects of endometriosis. I. Genetic studies. Am J Obstet Gynecol 1980; 137: 327-331.
22. Moen $\mathrm{NH}$, Magnus $\mathrm{P}$. The familial risk of endometriosis. Acta Obstet Gynecol Scand 1993; 72: 560-564.

23. Moen NH. Endometriosis in monozygotic twins. Acta Obstet Gynecol Scand 1994; 73: 59-62.

24. Kennedy S, Mardou H, Barlow D. Familial endometriosis. J Assist Reprod Genet 1995; 12: $32-34$

25. Kosugi Y, Elias S, Malinak LR, Nagata J, Isaka K, Takayama M, Simpson JL, Bischoft FZ. Increased heterogeneity of chromosome 17 aneuploidy in endometriosis. Am J Obstet Gynecol 1999; 80: 792-797.

26. Pallard S, Davies HFFS, Calue R. Perioperative appearance of serum class I antigen during liver transplantation. Transplantation 1990; 49: 659-663.

27. Athanassakis I, Paflis M, Ranella A, Vassiliadis S. Detection of soluble HLA$\mathrm{G}$ levels in maternal serum can be predictive for a successful pregnancy. Transplant Proc 1999; 31: 1834-1837.

28. Simpson JL, Malinak LR, Elias S, Carson SA, Radvany RA. HLA associations in endometriosis. Am J Obstet Gynecol 1984; 148: 395-397.

Received 8 January 2001; accepted 1 March 2001 


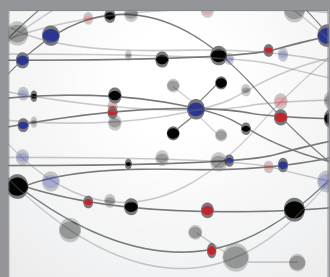

The Scientific World Journal
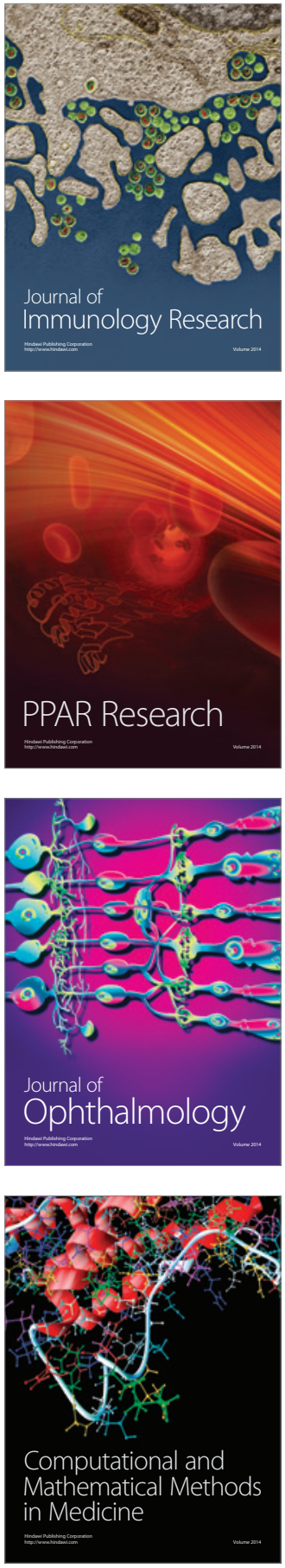

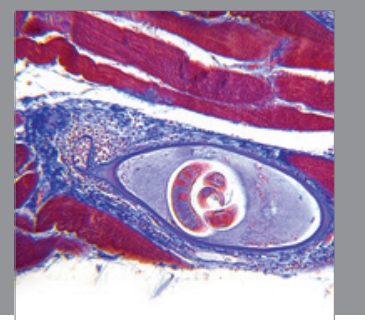

Gastroenterology

Research and Practice
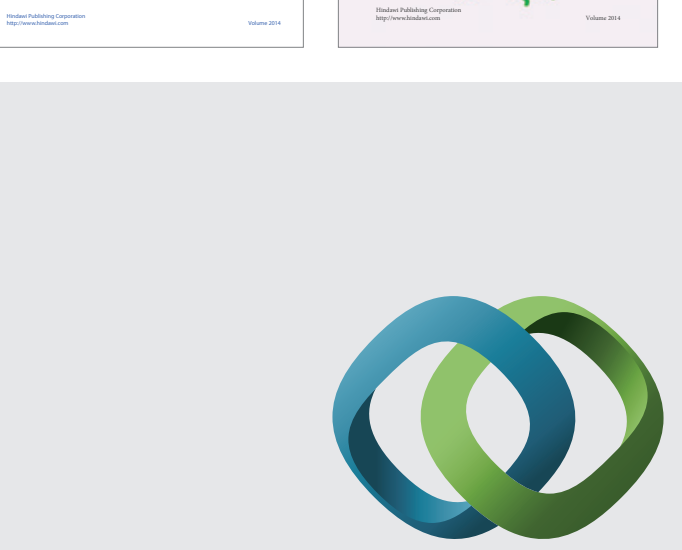

\section{Hindawi}

Submit your manuscripts at

http://www.hindawi.com
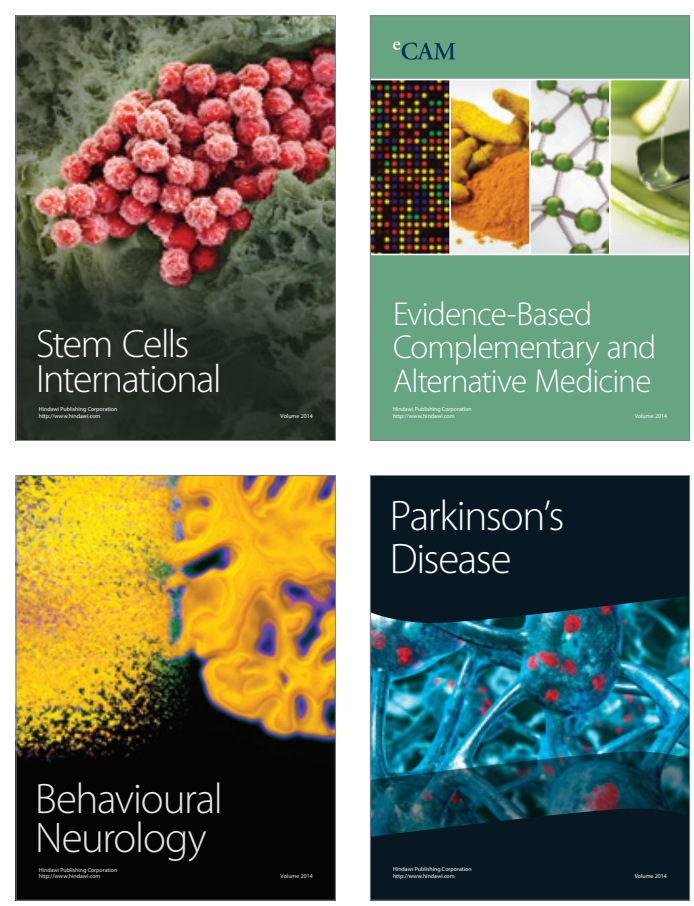

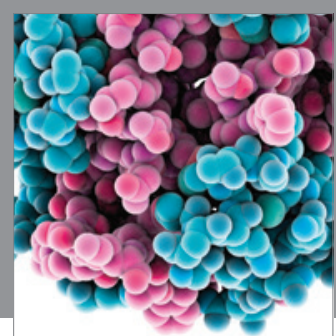

Journal of
Diabetes Research

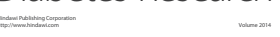

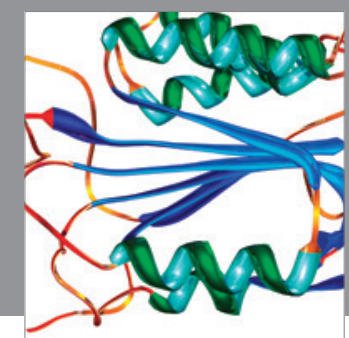

Disease Markers
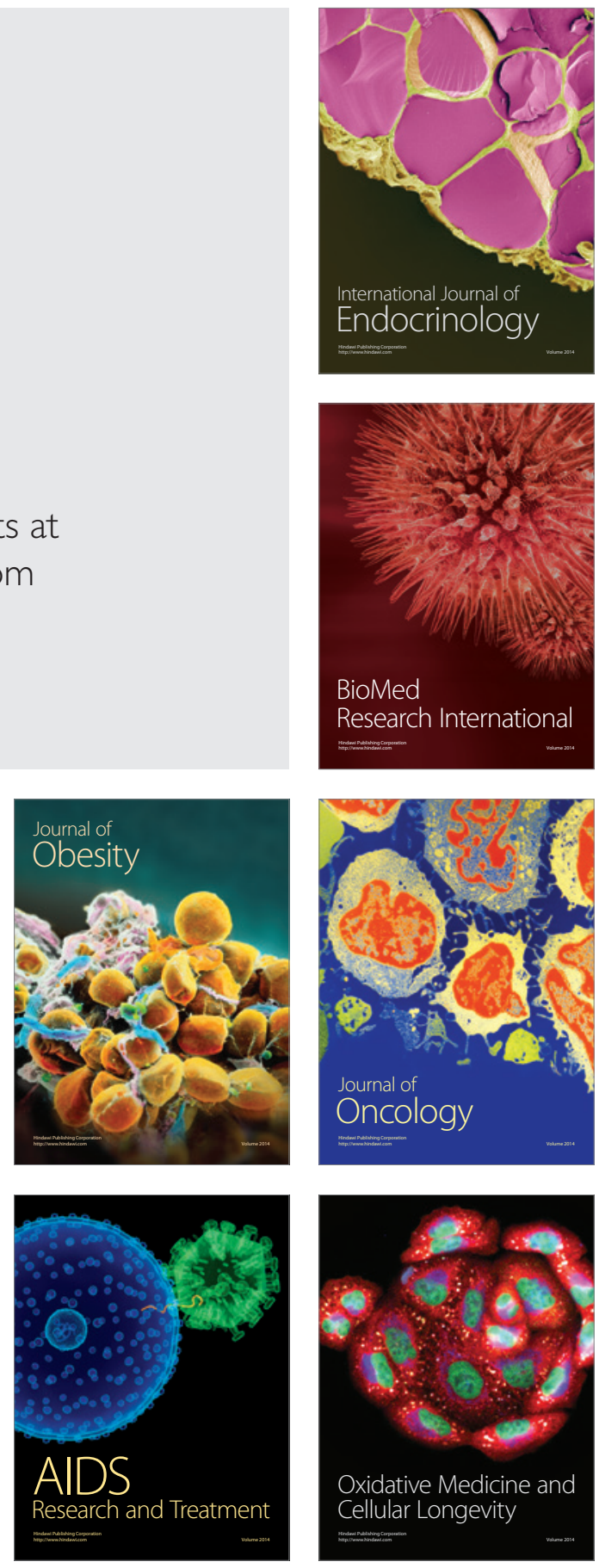